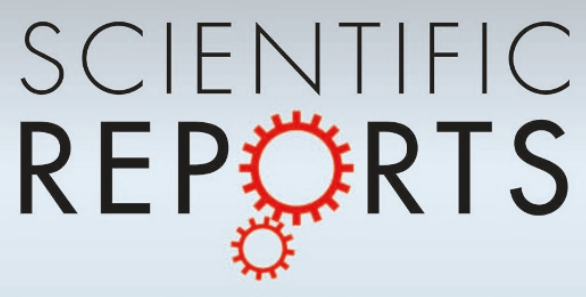

OPEN

SUBJECT AREAS:

ELECTRONIC DEVICES

SPINTRONICS

TOPOLOGICAL INSULATORS

ELECTRONIC PROPERTIES AND

MATERIALS

Received

14 March 2013

Accepted

23 July 2013

Published

12 August 2013

Correspondence and requests for materials should be addressed to X.J.Z. (XJZhou@aphy. iphy.ac.cn.)

\title{
Tunable Dirac Fermion Dynamics in Topological Insulators
}

Chaoyu Chen', Zhuojin Xie', Ya Feng', Hemian Yi', Aiji Liang', Shaolong He', Daixiang Mou', Junfeng He' ', Yingying Peng' ', Xu Liu', Yan Liu', Lin Zhao', Guodong Liu', Xiaoli Dong', Jun Zhang' ' Li Yu', Xiaoyang Wang ${ }^{2}$, Qinjun Peng' ${ }^{2}$, Zhimin Wang ${ }^{2}$, Shenjin Zhang ${ }^{2}$, Feng Yang ${ }^{2}$, Chuangtian Chen ${ }^{2}$, Zuyan Xu' \& X. J. Zhou'

${ }^{1}$ Beijing National Laboratory for Condensed Matter Physics, Institute of Physics, Chinese Academy of Sciences, Beijing 100190,
China, ${ }^{2}$ Technical Institute of Physics and Chemistry, Chinese Academy of Sciences, Beiijing 100190, China.

Three-dimensional topological insulators are characterized by insulating bulk state and metallic surface state involving relativistic Dirac fermions which are responsible for exotic quantum phenomena and potential applications in spintronics and quantum computations. It is essential to understand how the Dirac fermions interact with other electrons, phonons and disorders. Here we report super-high resolution angle-resolved photoemission studies on the Dirac fermion dynamics in the prototypical $\mathrm{Bi}_{2}(\mathrm{Te}, \mathrm{Se})_{3}$ topological insulators. We have directly revealed signatures of the electron-phonon coupling and found that the electron-disorder interaction dominates the scattering process. The Dirac fermion dynamics in $\mathrm{Bi}_{2}\left(\mathrm{Te}_{3-\mathrm{x}} \mathrm{Se}_{\mathrm{x}}\right)$ topological insulators can be tuned by varying the composition, $\mathrm{x}$, or by controlling the charge carriers. Our findings provide crucial information in understanding and engineering the electron dynamics of the Dirac fermions for fundamental studies and potential applications.

T opological insulators represent a new state of matter that has an insulating bulk state but a conducting surface/edge state which hosts an unique spin texture and a linear dispersion relation compatible with massless Dirac fermions $s^{1-3}$. In addition to their potential applications in spintronics and quantum computing ${ }^{4,5}$, the topological insulators also provide a promising platform for realizing a number of novel and exotic quantum phenomena including quantum spin Hall effect ${ }^{6}$, fractional charge and quantized current $^{7}$, dynamical axion field ${ }^{8}$, magnetic monopole ${ }^{9}$, Majorana fermions ${ }^{10,11}$ and quantum anomalous Hall effect ${ }^{12}$. Some of the phenomena have been observed experimentally in two-dimensional topological insulators, such as quantum spin Hall effect ${ }^{13}$ and quantum Hall effect ${ }^{14}$ in the HgTe quantum wells. However, in the case of three-dimensional topological insulators like $\mathrm{Bi}_{2}(\mathrm{Te}, \mathrm{Se})_{3}$ family ${ }^{15-18}$, in spite of intensive efforts from transport experiments ${ }^{19-23}$, attempts to observe electronic transport signatures of the surface Dirac fermions have been hampered by a couple of obstacles. First, contrary to usual expectation that the bulk is insulating in topological insulators, the presence of defects in $\mathrm{Bi}_{2}(\mathrm{Te}, \mathrm{Se})_{3}$ system makes the bulk conducting ${ }^{24}$ and the transport properties are then dominated by the bulk state instead of the desired surface state ${ }^{20}$. Second, in several cases where the surface transport signatures are identified, more than one frequency or one type of charge carriers contribute to the quantum oscillation ${ }^{21-23}$, indicating the complexity of sample surface exposed to atmosphere or protection layers ${ }^{25}$. Third, the transport mobility of charge carriers in the $\mathrm{Bi}_{2}(\mathrm{Te}, \mathrm{Se})_{3}$ topological insulators, $\mu^{\prime} \sim 10^{4} \mathrm{~cm}^{2} V^{-1} \mathrm{~s}^{-1}$ (Refs. 19,23,26) is not sufficiently high compared to those from HgTe quantum wells $\left(1.5 \times 10^{5} \mathrm{~cm}^{2} V^{-1} \mathrm{~s}^{-1}\right)^{13,14}$ and graphene $(2.3 \times$ $\left.10^{5} \mathrm{~cm}^{2} V^{-1} \mathrm{~s}^{-1}\right)^{27-29}$. Although strict electron backscattering is forbidden in topological insulators due to time reversal symmetry invariance, it is important to uncover other scattering channels that limit the transport mobility of the two-dimensional Dirac fermions ${ }^{26}$.

The charge transport properties are dictated by the electron dynamics in materials. In particular, the scattering of electrons by other entities such as other electrons, phonons and disorder, determines the lifetime of the quasiparticles, and thus the electron mobility and mean free path in the transport process ${ }^{30}$. Angle-resolved photoemission spectroscopy (ARPES) has become a powerful tool to directly probe such many-body effects ${ }^{31,32}$. Under the sudden approximation, ARPES measures a single particle spectral function

$$
A(k, \omega)=\frac{1}{\pi} \frac{|\operatorname{Im} \Sigma(k, \omega)|}{\left[\omega-\epsilon_{k}-\operatorname{Re} \Sigma(k, \omega)\right]^{2}+[\operatorname{Im} \Sigma(k, \omega)]^{2}}
$$


with $k$ and $\omega$ representing electron momentum and energy, respectively. The real and imaginary parts of electron self-energy, $\operatorname{Re} \Sigma(k, \omega)$ and $\operatorname{Im} \Sigma(k, \omega)$, carry all the information about the band renormalization and quasiparticle lifetime in an interacting system ${ }^{31,32}$.

There have been a few investigations on the dynamics of Dirac fermions in $\mathrm{Bi}_{2}(\mathrm{Te}, \mathrm{Se})_{3}$ topological insulators using $\mathrm{ARPES}^{33,34}$ or helium atom surface scattering probes ${ }^{35}$. However, the results obtained so far are not consistent. While the electron-phonon coupling strength $(\lambda)$ was reported to be 0.25 in $\mathrm{Bi}_{2} \mathrm{Se}_{3}$ from temperaturedependent photoemission measurements ${ }^{33}$, it was claimed that the electron-phonon coupling is exceptionally weak in $\mathrm{Bi}_{2} \mathrm{Se}_{3}$ in another measurement ${ }^{34}$. The inconsistency comes presumably from two issues related to the ARPES study on many-body effects in topological insulators. First, the coexistence of bulk bands and surface bands near the Fermi level, due to the bulk doping by defects, complicates the identification of weak band renormalization effects in the surface bands. Second, the small momentum space and energy window of the Dirac fermions and the weak many-body effects require high precision ARPES measurements. The momentum space occupied by the surface state, $\sim \pm 0.1 \AA^{-1}$ for $\mathrm{Bi}_{2} \mathrm{Se}_{3}$ (Fig. 1a) and $\sim \pm$ $0.04 \AA^{-1}$ for p-type $\mathrm{Bi}_{2} \mathrm{Te}_{3}$ (Fig. 2a), is rather small ${ }^{17,18,25}$. To overcome these problems, it is ideal to first suppress the interference of the bulk bands in the measurements. Since the as-grown $\mathrm{Bi}_{2} \mathrm{Se}_{3}$ is electron-doped (n-type) while $\mathrm{Bi}_{2} \mathrm{Te}_{3}$ can be hole-doped (p-type) ${ }^{25}$, balancing the proportion of selenium and tellurium in $\mathrm{Bi}_{2}(\mathrm{Se}, \mathrm{Te})_{3}$ system may neutralize the bulk carrier doping and reduce the effect of the bulk bands on the surface state. Furthermore, vacuum ultraviolet (VUV) laser-based ARPES ${ }^{36}$ can help suppress the bulk bands ${ }^{25}$, in addition to its super-high energy and momentum resolutions (Supplementary Fig. S2) that are desirable in studying manybody effects in topological insulators.

\section{Results}

Signatures of electron-phonon coupling in $\mathrm{Bi}_{2} \mathrm{Se}_{3}$. The $\mathrm{Bi}_{2} \mathrm{Se}_{3}$ sample shows sharp surface state band (Fig. 1a) that facilitates the investigation of the Dirac fermion dynamics in the material. The momentum distribution curves (MDCs) at different binding energies (Fig. 1b) show well-defined sharp peaks that can be fitted by Lorentzian lineshape to obtain peak position and peak width (Full-Width-at-Half-Maximum, $\Gamma_{M D C}$ ) (Supplementary Fig. S3). The obtained MDC peak position as a function of the binding energy gives the measured dispersion relation (left panels of Fig. 1c and $1 \mathrm{~d}$ ). We assume a bare band as a linear line that connects the two points on the measured dispersion, one at the Fermi level and the other at $100 \mathrm{meV}$ binding energy. This is reasonable within such a small energy window and particularly for the Dirac fermions that shows a linear dispersion over a large energy range. The effective real part of the electron self-energy $(\operatorname{Re} \Sigma)$ is obtained by subtracting the measured dispersion with the bare band, as shown in right panels of Fig. 1c and Fig. 1d (black empty circles). The imaginary part of the electron self-energy $(\operatorname{Im} \Sigma)$ is obtained from the fitted MDC width: $\operatorname{Im} \Sigma=\Gamma_{M D C} \times v_{0} / 2$ with $v_{0}$ being the Fermi velocity of the bare band (right panels of Fig. 1c and Fig. 1d, blue empty squares) ${ }^{32}$.

The extraction of the electron self-energy provides full information for understanding the electron dynamics in a material where the electron scattering usually involves three main processes: electron-electron interaction, electron-phonon coupling, and electron-disorder interaction ${ }^{37,38}$. The electron-disorder interaction is characterized by the imaginary part of the electron self-energy at zero energy (Fermi level) since the contribution from the electronelectron interaction and electron-phonon coupling approaches zero at the zero energy at very low temperature. The electron-phonon interaction is characterized by the band renormalization (usually a kink in dispersion, corresponding to a peak in the real part of the electron self-energy) and a drop in the imaginary part of the electron self-energy. As exemplified in Fig. 1 for $\mathrm{Bi}_{2} \mathrm{Se}_{3}$, there are clear indications of electron-phonon coupling revealed, such as the kink in dispersions (left panels of Fig. 1c and Fig. 1d), peak in the real part of the electron self-energy and a drop in the imaginary part of the electron self-energy (right panels of Fig. 1c and Fig. 1d). The characteristic energy scale of the phonons involved $\left(\omega_{0}\right)$ is determined by the peak position of the real part of the electron self-energy; in the case of $\mathrm{Bi}_{2} \mathrm{Se}_{3}$, it is $\sim 18 \mathrm{meV}$ as marked by arrows in Fig. 1c and $1 \mathrm{~d}$. The electron-phonon coupling strength, $\lambda$, can be determined by the
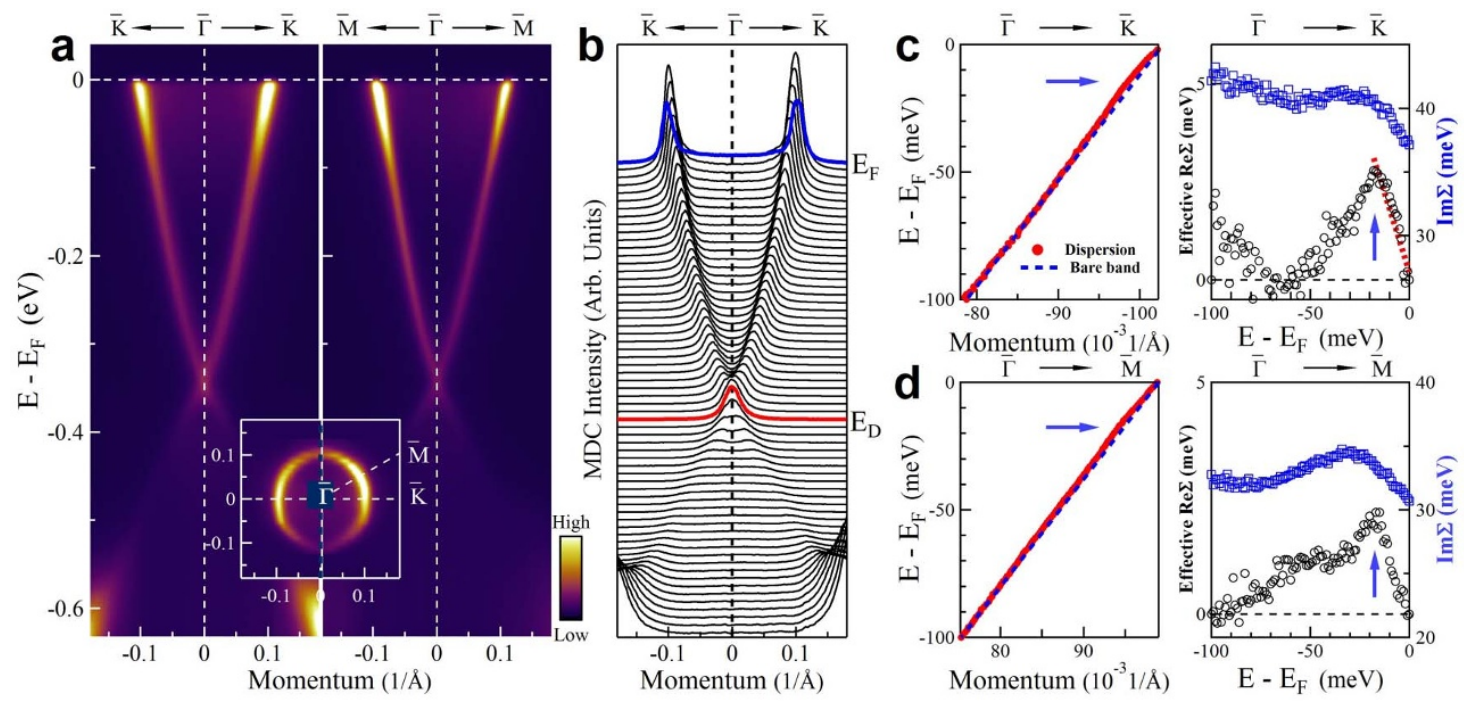

Figure 1 Observation of electron-phonon coupling in $\mathrm{Bi}_{2} \mathrm{Se}_{3}$. (a) Surface state dispersions along $\bar{\Gamma}-\bar{K}$ (left panel) and $\bar{\Gamma}-\bar{M}($ right panel) directions. The inset shows the Fermi surface of $\mathrm{Bi}_{2} \mathrm{Se}_{3}$. (b) Momentum distribution curves (MDCs) of the surface state dispersion along $\bar{\Gamma}-\bar{K}$. Red line and blue line indicate the MDCs at the Dirac point $\left(\mathrm{E}_{D}\right)$ and the Fermi level $\left(\mathrm{E}_{F}\right)$, respectively. (c) Dispersion and effective electron self-energy of surface state band along $\bar{\Gamma}-\bar{K}$ direction. The left panel shows the measured dispersion (red circles) obtained from MDC fitting. The blue dotted line represents an empirical bare band that is a straight line connecting two points in the measured dispersion, one at $\mathrm{E}_{F}$ and the other at $100 \mathrm{meV}$ binding energy. The right panel shows the effective real part of the electron self-energy, $\operatorname{Re} \Sigma(k, \omega)$ (black circles), and imaginary part of the electron self-energy, $\operatorname{Im} \Sigma(k, \omega)$ (blue squares). The red dashed line is a linear fit to the low-energy region of $\operatorname{Re} \Sigma(k, \omega)$. Blue arrows indicate the characteristic energy scale of the electronphonon coupling. (d)Dispersion and effective electron self-energy of surface state band along $\bar{\Gamma}-\bar{M}$ direction. 

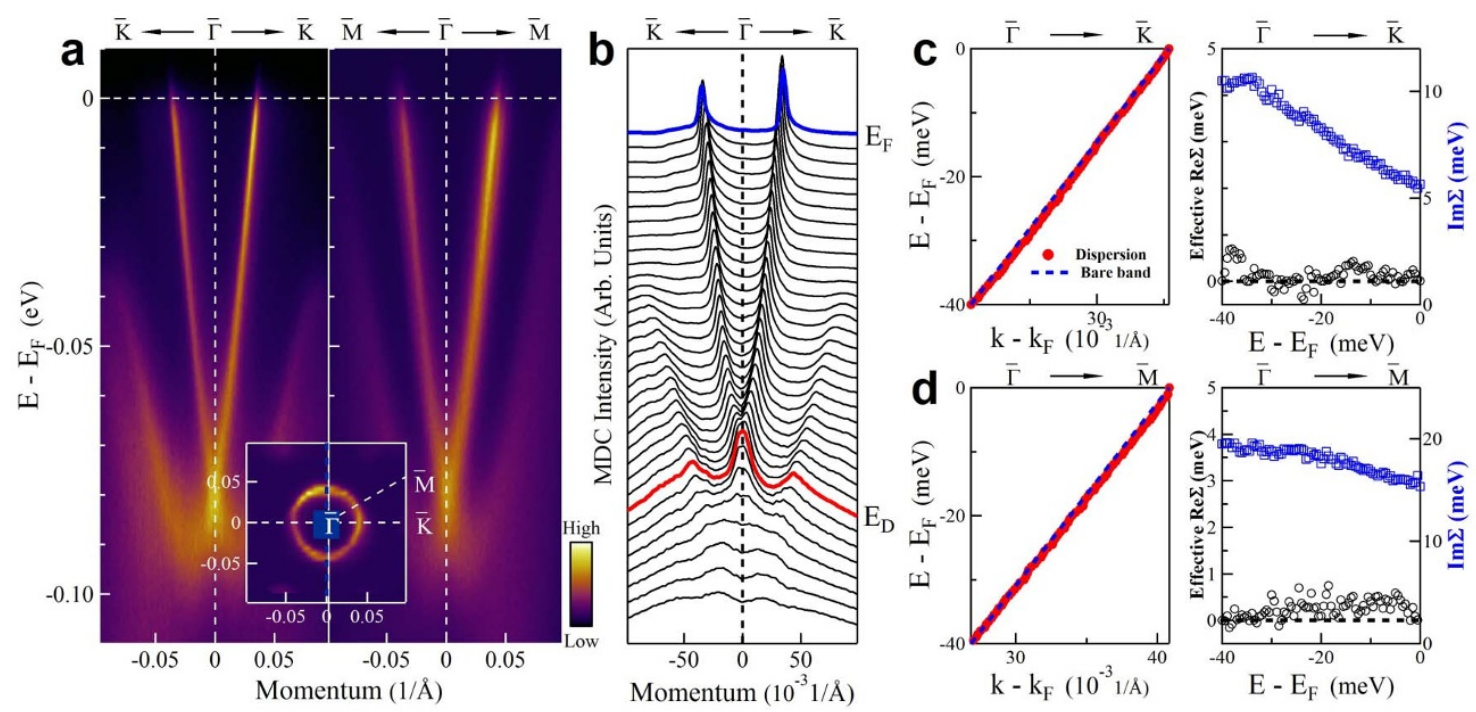

Figure $2 \mid$ Extremely weak electron-phonon coupling in p-type $\mathbf{B i}_{2} \mathbf{T e}_{3}$. (a) Surface state dispersions along $\bar{\Gamma}-\bar{K}$ (left panel) and $\bar{\Gamma}-\bar{M}$ (right panel) directions. The inset shows the Fermi surface of p-type $\mathrm{Bi}_{2} \mathrm{Te}_{3}$. (b) Momentum distribution curves (MDCs) of the surface state dispersion along $\bar{\Gamma}-\bar{K}$. Red line and blue line indicate the MDCs at the Dirac point $\left(\mathrm{E}_{D}\right)$ and the Fermi level $\left(\mathrm{E}_{F}\right)$, respectively. (c) Dispersion and effective electron self-energy of surface state band along $\bar{\Gamma}-\bar{K}$ direction. The left panel shows the measured dispersion (red circles) obtained from MDC fitting. The blue dotted line represents an empirical bare band that is a straight line connecting two points in the measured dispersion, one at $\mathrm{E}_{F}$ and the other at $40 \mathrm{meV}$ binding energy. The right panel shows the effective real part of the electron self-energy, $\operatorname{Re} \Sigma(k, \omega)$ (black circles), and imaginary part of the electron self-energy, $\operatorname{Im} \Sigma(k, \omega)$ (blue squares). (d)Dispersion and effective electron self-energy of surface state band along $\bar{\Gamma}-\bar{M}$ direction.

slope of the line near the zero energy in the real part of the electron self-energy. As shown by red dashed line in the right panel of Fig. 1c, it is determined to be $\lambda \sim 0.17$ for $\mathrm{Bi}_{2} \mathrm{Se}_{3}$. The electron-electron interaction manifests itself more clearly in the imaginary part of the electron self-energy. In a typical Fermi liquid case, its contribution is proportional to the $\left(\mathrm{E}-\mathrm{E}_{F}\right)^{2}: \mathrm{Im}_{e e}=\beta\left(\mathrm{E}-\mathrm{E}_{F}\right)^{2}$. Since the electronphonon contribution is constant above the characteristic phonon scale, the deviation from the constant above the phonon scale gives a measure of the electron-electron interaction involved. For $\mathrm{Bi}_{2} \mathrm{Se}_{3}$ and the $\mathrm{Bi}_{2}(\mathrm{Se}, \mathrm{Te})_{3}$ series, we find that the electron-electron contribution is weak $\left(\beta \sim 0.014\right.$ for $\left.\mathrm{Bi}_{2} \mathrm{Se}_{3}\right)$, particularly when compared with the dominant electron-disorder interaction and the electronphonon coupling. Therefore, in the following, we will mainly focus on the electron-phonon coupling and electron-disorder interaction.

As shown in Fig. 1, our measurements clearly indicate that there is an obvious electron-phonon coupling $(\lambda \sim 0.17)$ in the electron dynamics of the Dirac fermions in $\mathrm{Bi}_{2} \mathrm{Se}_{3}$. It is nearly isotropic because similar behaviors are observed along the $\bar{\Gamma}-\bar{K}$ (Fig. 1c) and $\bar{\Gamma}-\bar{M}$ (Fig. 1d) momentum cuts. The characteristic energy scale, $\sim 18 \mathrm{meV}$, is consistent with the highest-frequency phonon mode $\left(A_{\mathrm{lg}}^{2}\right)$ at $\sim 20 \mathrm{meV}$ in $\mathrm{Bi}_{2} \mathrm{Se}_{3}$ from both theoretical calculations ${ }^{39}$ and Raman measurements ${ }^{40}$ (Supplementary Fig. S7). This is in strong contrast to a recent report where exceptionally weak electron-phonon coupling was claimed and no dispersion kink was resolved in $\mathrm{Bi}_{2} \mathrm{Se}_{3}{ }^{34}$. We believe that the clear manifestation of electron-phonon coupling in our measurements is most likely due to much improved instrumental resolution we have achieved.

Extremely weak electron-phonon coupling in p-type $\mathbf{B i}_{2} \mathbf{T e}_{3}$. The electron-phonon coupling is extremely weak in the p-type $\mathrm{Bi}_{2} \mathrm{Te}_{3}$ sample, as seen in Fig. 2. This sample was prepared under similar condition as the above $\mathrm{Bi}_{2} \mathrm{Se}_{3}$ sample and it is p-type because the Fermi level intersects with the bulk valence band ${ }^{25}$. Even measured with such a super-high instrumental resolution (Supplementary Fig. S2), we do not resolve any clear signature of electron-phonon coupling. The measured dispersion is basically a straight line, corresponding to a real part of electron self-energy that is nearly zero (black empty circles in right panels of Fig. $2 \mathrm{c}$ and Fig. 2d).
There is no clear drop in the imaginary part of the electron selfenergy (blue empty squares in right panels of Fig. 2c and Fig. 2d). All these observations point to an extremely weak electron-phonon coupling in the p-type $\mathrm{Bi}_{2} \mathrm{Te}_{3}$ sample. In the mean time, the p-type $\mathrm{Bi}_{2} \mathrm{Te}_{3}$ also exhibits a rather weak electron-disorder scattering when compared with $\mathrm{Bi}_{2} \mathrm{Se}_{3}$. As seen from the right panel of Fig. $2 c$, the imaginary part of the electron self-energy at zero energy is only $5.6 \mathrm{meV}$ along the $\bar{\Gamma}-\bar{K}$ direction. This is significantly lower than $37 \mathrm{meV}$ along the same direction in $\mathrm{Bi}_{2} \mathrm{Se}_{3}$ topological insulator (right panel of Fig. 1c).

Tunability of Dirac fermion dynamics in the $\mathrm{Bi}_{2}\left(\mathrm{Te}_{3-x} \mathrm{Se}_{x}\right)$ series. The dramatically different behaviors between $\mathrm{Bi}_{2} \mathrm{Se}_{3}$ (Fig. 1) and p-type $\mathrm{Bi}_{2} \mathrm{Te}_{3}$ (Fig. 2) motivated us to further investigate the composition dependence of the Dirac fermion dynamics in the $\mathrm{Bi}_{2}\left(\mathrm{Te}_{3-x} \mathrm{Se}_{x}\right)$ series. We observe a systematic evolution of band structure, electron-phonon coupling, and electron-disorder scattering, with the change of composition $\mathrm{x}$ in $\mathrm{Bi}_{2}\left(\mathrm{Te}_{3-x} \mathrm{Se}_{x}\right)$ (Fig. 3). Note that all these samples are prepared under similar conditions. First, the location of the Dirac point $\left(\mathrm{E}_{D}\right)$ moves monotonically to higher binding energy with increasing $x$. For $0 \leq$ $x \leq 0.05$, the samples are p-type because the Fermi level intersects with the bulk valence band. For $0.15 \leq x<1.5$, the Fermi level intersects with the surface state bands only. For $1.5 \leq x \leq 3$, the samples become n-type because the Fermi level intersects with the bulk conduction band. Note that the Dirac point exhibits a big jump when $x$ changes from 0.05 to 0.15 . Second, there is an overall increase of electron-disorder interaction with increasing $\mathrm{x}$ in $\mathrm{Bi}_{2}\left(\mathrm{Te}_{3-x} \mathrm{Se}_{x}\right)$. This can be seen from the increase of the imaginary part of the electron self-energy and hence its value at zero energy with increasing $\mathrm{x}$. This indicates that the $\mathrm{Bi}_{2}\left(\mathrm{Te}_{3-x} \mathrm{Se}_{x}\right)$ system gets more disordered with increasing $\mathrm{x}$. Third, there is also an overall increase in the electron-phonon coupling strength with increasing $\mathrm{x}$ in $\mathrm{Bi}_{2}\left(\mathrm{Te}_{3-x} \mathrm{Se}_{x}\right)$. Although no indication of electron-phonon coupling is observed in p-type $\mathrm{Bi}_{2} \mathrm{Te}_{3}$, a slight substitution of Te by Se, with $\mathrm{x}=0.05$, already renders a clear observation of electronphonon coupling in $\mathrm{Bi}_{2}\left(\mathrm{Te}_{2.95} \mathrm{Se}_{0.05}\right)$, as seen in the appearance of a peak in the real part of the electron self-energy (black circles in 

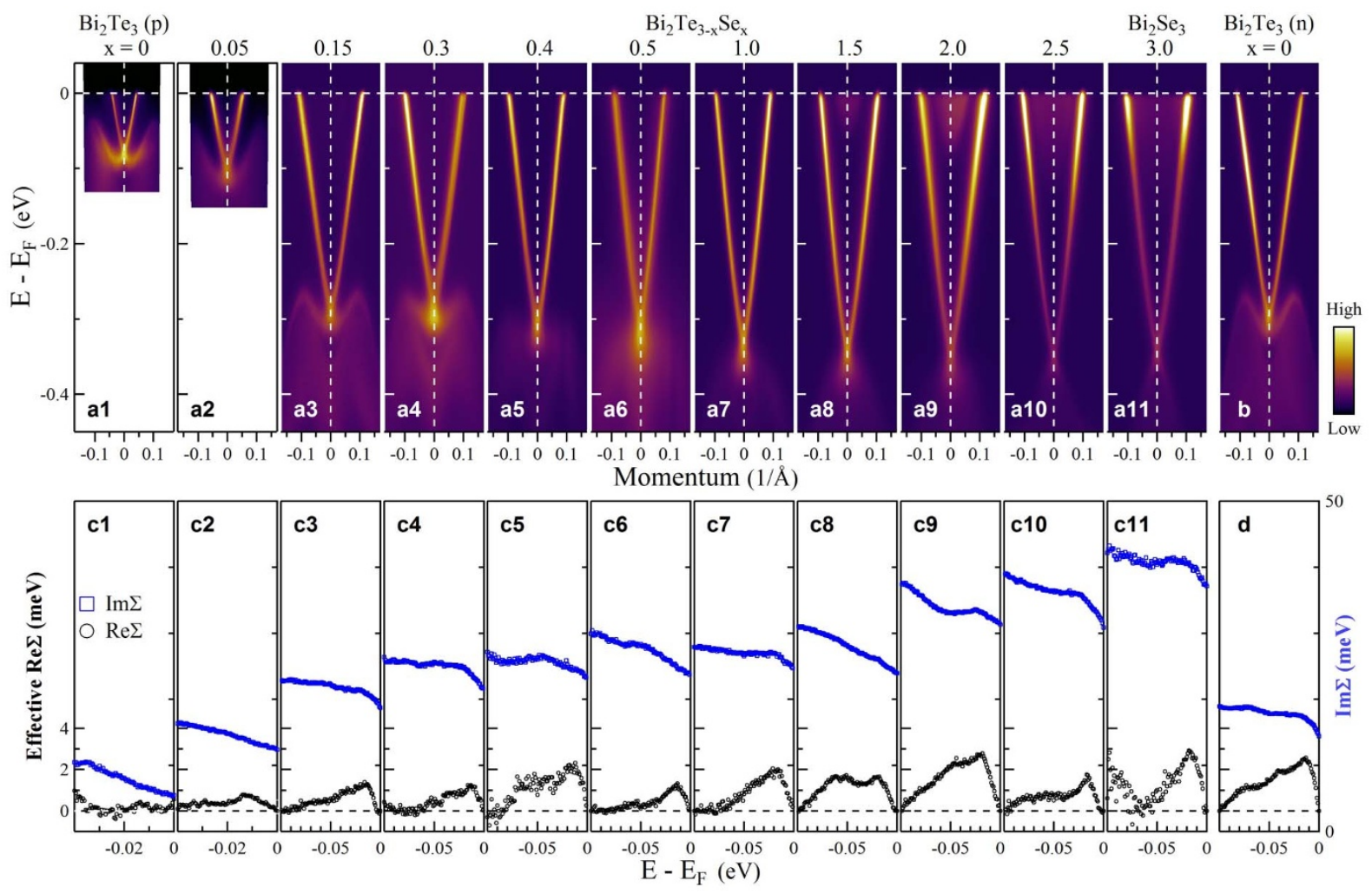

Figure 3 Evolution of surface state band structure and electron self-energy with the composition, $x$, in $\mathrm{Bi}_{2}\left(\mathrm{Te}_{3-x} \mathrm{Se}_{x}\right)$ system. (a) The surface state band structure of $\mathrm{Bi}_{2} \mathrm{Te}_{3-x} \mathrm{Se}_{x}$ with various compositions, $\mathrm{x}$, measured along the $\bar{\Gamma}-\overline{\mathrm{K}}$ direction. (b) The surface state band structure of $\mathrm{n}$-type $\mathrm{Bi}_{2} \mathrm{Te}_{3}$ along the $\bar{\Gamma}-\bar{K}$ direction. (c) Corresponding effective real part of the electron self-energy (black circles) and imaginary part of the electron self-energy (blue squares) for $\mathrm{Bi}_{2}\left(\mathrm{Te}_{3}-\mathrm{Se}_{x}\right.$ ) with different compositions. (d) Corresponding effective real part of the electron self-energy (black circles) and imaginary part of the electron self-energy (blue squares) for $\mathrm{n}$-type $\mathrm{Bi}_{2} \mathrm{Te}_{3}$.

Fig. $3 \mathrm{c} 2$ ). The electron-phonon coupling strength increases from nearly zero for p-type $\mathrm{Bi}_{2} \mathrm{Te}_{3}$ to $\lambda \sim 0.17$ for $\mathrm{Bi}_{2} \mathrm{Se}_{3}$.

It is interesting that for the same $\mathrm{Bi}_{2} \mathrm{Te}_{3}$ nominal composition, when prepared under different conditions, samples can become either p-type or n-type that exhibit dramatic difference in their Dirac fermion dynamics. As shown in Fig. 3b, the Dirac point of the n-type $\mathrm{Bi}_{2} \mathrm{Te}_{3}$ lies nearly $310 \mathrm{meV}$ below the Fermi level which differs significantly from the $85 \mathrm{meV}$ location in the p-type $\mathrm{Bi}_{2} \mathrm{Te}_{3}$ (Fig. 3a1). Particularly, the n-type $\mathrm{Bi}_{2} \mathrm{Te}_{3}$ exhibits a pronounced electron-phonon coupling, as seen from the obvious peak in the real part of the electron self-energy (black circles in Fig. 3d) and a drop in the imaginary part of the electron self-energy (blue squares in Fig. 3d). The resultant electron-phonon coupling constant is $\lambda \sim 0.19$, which is even slightly larger than that in $\mathrm{Bi}_{2} \mathrm{Se}_{3}(\lambda \sim 0.17)$. The electron-disorder scattering also increases in the n-type $\mathrm{Bi}_{2} \mathrm{Te}_{3}$ compared to the p-type one, as the value of the imaginary part of the electron self energy at zero energy becomes much larger (Fig. 3d). However, compared with $\mathrm{Bi}_{2} \mathrm{Se}_{3}$, the disorder scattering in n-type $\mathrm{Bi}_{2} \mathrm{Te}_{3}$ remains much weaker(Fig. $3 \mathrm{c} 11$ ). The first principle calculation, which expects a rather weak electron-phonon coupling in $\mathrm{Bi}_{2} \mathrm{Te}_{3}(\lambda \sim 0.05)^{41}$, is hard to explain the strong electron-phonon coupling in n-type $\mathrm{Bi}_{2} \mathrm{Te}_{3}$ and particularly the disparity of electron-phonon coupling between the n-type and p-type $\mathrm{Bi}_{2} \mathrm{Te}_{3}$.

The different electron dynamics between $\mathrm{Bi}_{2} \mathrm{Se}_{3}$ and $\mathrm{Bi}_{2} \mathrm{Te}_{3}$, and between $n$-type $\mathrm{Bi}_{2} \mathrm{Te}_{3}$ and p-type $\mathrm{Bi}_{2} \mathrm{Te}_{3}$, is intriguing. We note that the native majority defect types are totally different in these samples, i.e., Se vacancies in $\mathrm{Bi}_{2} \mathrm{Se}_{3}$, Te-Bi antisites in the $\mathrm{n}$-type $\mathrm{Bi}_{2} \mathrm{Te}_{3}$, and $\mathrm{Te}$ vacancies in the p-type $\mathrm{Bi}_{2} \mathrm{Te}_{3}$. The difference of the electron-disorder interaction can be due to such different types of disorders and their densities in the samples. In addition, as the main difference between the $n$-type and p-type $\mathrm{Bi}_{2} \mathrm{Te}_{3}$ lies mainly in the relative position between the Fermi level and the bulk bands, it is reasonable to deduce that the bulk bands may play a role in the electron dynamics of the Dirac fermions. This is consistent with the observation that, for $n$-type $\mathrm{Bi}_{2} \mathrm{Te}_{3}(\mathrm{x}=0)$, although its composition is rather different from $\mathrm{Bi}_{2}\left(\mathrm{Te}_{2.85} \mathrm{Se}_{0.15}\right)(\mathrm{x}=0.15)$, their Dirac fermion dynamics is quite similar in both the electron-phonon coupling and electron-disorder interaction (Figs. 3 and 4). The distinction of the electron-phonon coupling between the n-type and p-type $\mathrm{Bi}_{2} \mathrm{Te}_{3}$ indicates that the control of the charge carrier doping can be an alternative way to tune the Dirac fermion dynamics in topological insulators.

One may wonder whether the origin of the observed inelastic scattering in $\mathrm{Bi}_{2}(\mathrm{Se}, \mathrm{Te})_{3}$-based topological insulators is related to the materials themselves (defect, atomic arrangement and etc.) or the intrinsic topological properties associated with the time reversal symmetry (TRS) invariance. The TRS-protection may reduce the scattering strength because strict backscattering is not allowed, but it is difficult to quantitatively analyze this weakening effect. Note that the entire $\mathrm{Bi}_{2}(\mathrm{Te}, \mathrm{Se})_{3}$ series are topological insulators. Therefore, we believe the systematic evolution of their electron dynamics is mainly a manifestation of the usual many-body effects, other than a manifestation of the intrinsic TRS-related topological properties.

Fig. 4 gives a quantitative summary of the electron-phonon coupling and electron-disorder scattering in the $\mathrm{Bi}_{2}\left(\mathrm{Te}_{3-x} \mathrm{Se}_{x}\right)$ system. The relative position between the Dirac point and the bulk band is depicted in Fig. $4 \mathrm{a}$ for a couple of typical compositions and both $\mathrm{p}$ and n-type $\mathrm{Bi}_{2} \mathrm{Te}_{3}$. With increasing $\mathrm{x}$ in $\mathrm{Bi}_{2}\left(\mathrm{Te}_{3-x} \mathrm{Se}_{x}\right)$, there is an abrupt jump in the electron-phonon coupling strength from $\mathrm{x}=0$ to $\mathrm{x}=0.15$, accompanied by a transition from $\mathrm{p}$-type to $\mathrm{n}$-type. Further increase of $\mathrm{x}$ leads to an overall slight increase of electron-phonon coupling (Fig. 4c). Meanwhile, the characteristic energy scale, $\omega_{0}$, 


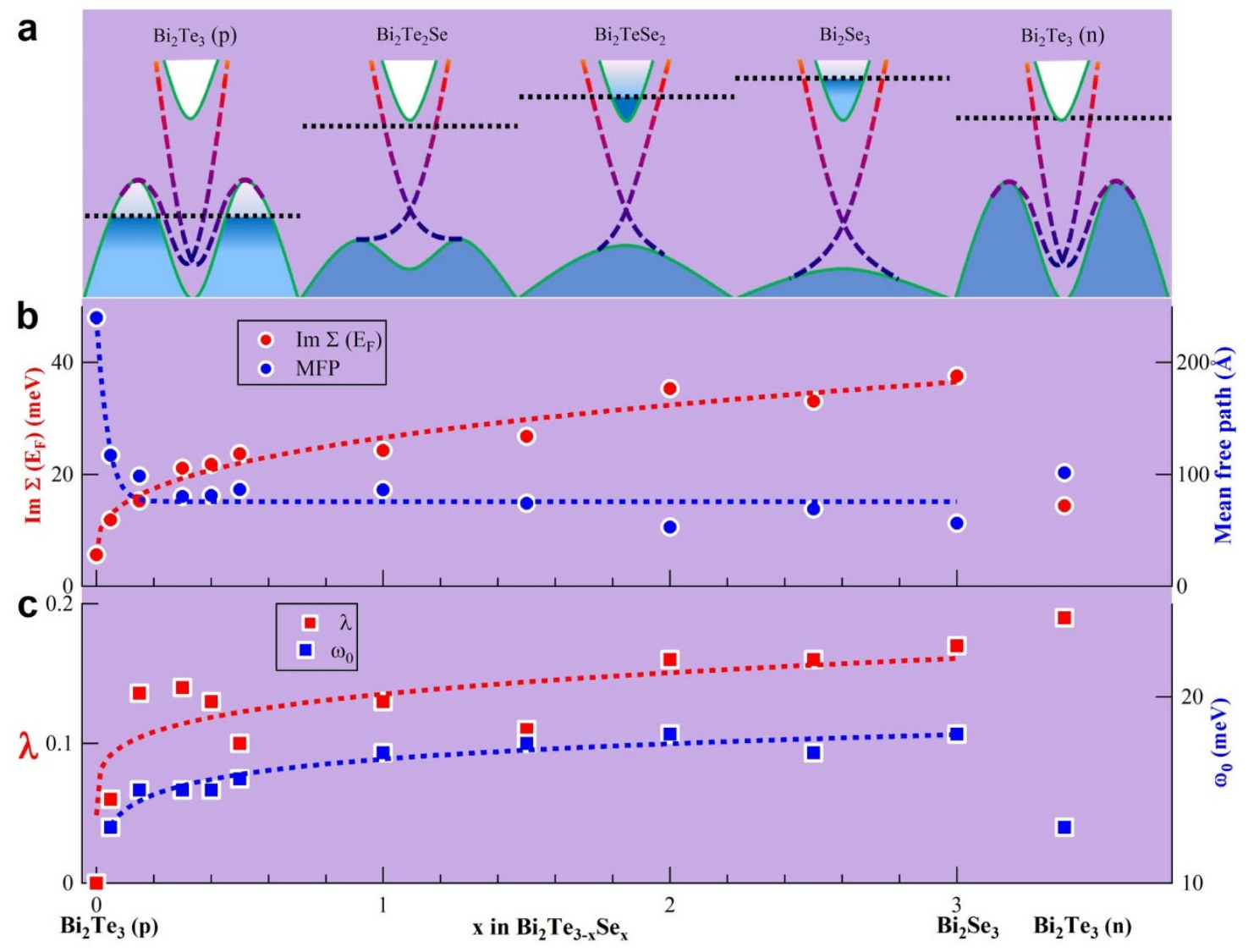

Figure 4 | Variation of electron-phonon coupling and electron-disorder scattering with composition, $x$, in $\mathrm{Bi}_{2}\left(\mathrm{Te}_{3-x} \mathrm{Se}_{x}\right)$ topological insulators. On the far right side, data on $\mathrm{n}$-type $\mathrm{Bi}_{2} \mathrm{Te}_{3}$ are also included. (a) Schematic band structure at several typical compositions to show the relative position between the topological surface states (color dashed lines) and the bulk states (green solid lines). Black dashed lines indicate the Fermi level. (b) Variation of the electron-disorder scattering (red circles) with composition, $\mathrm{x}$, in $\mathrm{Bi}_{2}\left(\mathrm{Te}_{3-} \mathrm{Se}_{x}\right)$ topological insulators. It is obtained by taking the value of the imaginary part of the electron self-energy at zero energy $\left(\mathrm{E}_{F}\right)$ as shown in Fig. 3c. Also shown is the electron mean free path (blue circles) calculated from the measured MDC width at the Fermi level. (c) Variation of the electron-phonon coupling strength $\lambda$ (red squares) and characteristic energy scale $\omega_{0}$ with the composition, $\mathrm{x}$, in $\mathrm{Bi}_{2}\left(\mathrm{Te}_{3-x} \mathrm{Se}_{x}\right)$ topological insulators and in n-type $\mathrm{Bi}_{2} \mathrm{Te}_{3}$. The dashed lines in (b) and (c) are guide to the eyes.

also increases with $\mathrm{x}$, which is consistent with the increase of the phonon frequency from $\mathrm{Bi}_{2} \mathrm{Te}_{3}$ to $\mathrm{Bi}_{2} \mathrm{Se}_{3}$ (Supplementary Fig. S7). This indicates that the $(\mathrm{Te}, \mathrm{Se})$ vibrations play a major role in dictating the electron-phonon coupling strength in the $\mathrm{Bi}_{2}\left(\mathrm{Te}_{3-x} \mathrm{Se}_{x}\right)$ system. This is also consistent with the observation that the characteristic energy scale is identical in n-type and p-type $\mathrm{Bi}_{2} \mathrm{Te}_{3}$ because their phonon frequencies are quite similar (Supplementary Fig. S7) although they have different electron-phonon coupling strength. It is clear that electron-disorder scattering is dominant in the scattering process. As seen from Fig. $4 \mathrm{~b}$, the imaginary part of the electron selfenergy at zero energy $\operatorname{Im} \Sigma\left(\mathrm{E}_{F}\right)$, which gives a good measure of the electron scattering rate, shows an overall increase from the p-type $\mathrm{Bi}_{2} \mathrm{Te}_{3}$ for $\mathrm{x}=0$ to $\mathrm{Bi}_{2} \mathrm{Se}_{3}$ for $\mathrm{x}=3$ in the $\mathrm{Bi}_{2}\left(\mathrm{Te}_{3-x} \mathrm{Se}_{x}\right)$ system (red circles in Fig. $4 \mathrm{~b}$ ). The quasi-particle mean free path, $\ell^{\prime}$, which can be determined from the MDC width $\Gamma_{M D C}, \ell^{\prime}=1 / \Gamma_{M D C}$, first decreases abruptly from the p-type $\mathrm{Bi}_{2} \mathrm{Te}_{3}(\mathrm{x}=0)$ to $\mathrm{x}=0.15$, followed by an overall slight decrease with $\mathrm{x}$ in $\mathrm{Bi}_{2}\left(\mathrm{Te}_{3-x} \mathrm{Te}_{x}\right)$ (blue circles in Fig. $3 \mathrm{~b})$. The electron mobility of the Dirac fermions $\left(\mu^{\prime}\right)$ is directly related to the quasi-particle mean free path, $\mu^{\prime}=e \ell^{\prime} /\left(\hbar k_{F}\right)$, with $\mathrm{k}_{F}$ being the Fermi momentum. It is then estimated that the electron mobility in p-type $\mathrm{Bi}_{2} \mathrm{Te}_{3}$ is near $1000 \mathrm{~cm}^{2} V^{-1} \mathrm{~s}^{-1}$ (Supplementary Fig. S4). By comparison, the electron mobility in $\mathrm{Bi}_{2} \mathrm{Se}_{3}$ and n-type $\mathrm{Bi}_{2} \mathrm{Te}_{3}$ is $\sim 70$ and $\sim 100 \mathrm{~cm}^{2} V^{-1} \mathrm{~s}^{-1}$, respectively, which are significantly lower than that of p-type $\mathrm{Bi}_{2} \mathrm{Te}_{3}$. We note that, although the electron mobility obtained from ARPES is not strictly the same as the electron mobility in the transport properties, they have good corres- pondence, especially for materials with circular Fermi surface and nearly isotropic electron scattering.

\section{Discussion}

The present study will provide important information for engineering the Dirac fermion dynamics in $\mathrm{Bi}_{2}\left(\mathrm{Te}_{3-x} \mathrm{Se}_{x}\right)$ topological insulators. It clearly indicates the presence of electron-phonon coupling and dominant role of the electron-disorder scattering in dictating the Dirac fermion dynamics in the system. The Dirac fermion dynamics can be tuned by varying either the composition or the charge carrier concentration. Specifically for the $\mathrm{Bi}_{2}\left(\mathrm{Te}_{3-x} \mathrm{Se}_{x}\right)$ system, our results clearly show that $\mathrm{Bi}_{2} \mathrm{Te}_{3}$ system is superior over $\mathrm{Bi}_{2} \mathrm{Se}_{3}$ in achieving higher electron mobility both due to its weak electron-phonon coupling and its weak electron-defect scattering. To further enhance the electron mobility in searching for novel quantum phenomena, it is necessary to choose a system with weak electron-phonon coupling and reduced disorder scattering.

\section{Methods}

High quality single crystals of $\mathrm{Bi}_{2}\left(\mathrm{Te}_{3-x} \mathrm{Se}_{x}\right)(0 \leq x \leq 3)$ were grown by the self-flux method. The mixed materials were heated to $1000^{\circ} \mathrm{C}$, held for 12 hours and then slowly cooled down to $500^{\circ} \mathrm{C}$ over 100 hours before cooling to room temperature. Single crystals of nearly one centimeter in size were obtained by cleaving. The angleresolved photoemission measurements were carried out on our vacuum ultra-violet (VUV) laser-based angle-resolved photoemission system, with an overall energy resolution of $\sim 1 \mathrm{meV}$. The momentum resolution is $\sim 0.004 \AA^{-1}$ for $30^{\circ}$ angular mode and $\sim 0.002 \AA^{-1}$ for $14^{\circ}$ angular mode of the R4000 electron energy analyzer. The samples were all cleaved and measured in vacuum with a base pressure better 
than $5 \times 10^{-11}$ Torr and a temperature $\mathrm{T} \sim 20 \mathrm{~K}$. For more details about the experimental methods, see supplementary information.

1. Hasan, M. Z. \& Kane, C. L. Colloquium:Topological insulators. Rev. Mod. Phys. 82, 3045-3067 (2010).

2. Moore, J. E. The birth of topological insulators. Nature 464, 194-198 (2010).

3. Qi, X. L. \& Zhang, S. C. Topological insulators and superconductors. Rev. Mod. Phys. 83, 1057-1110 (2011).

4. Qi, X. L. \& Zhang, S. C. The quantum spin Hall effect and topological insulators. Phys. Today 63, 33-38 (2010).

5. Moore, J. Topological insulators the next generation. Nat. Phys. 5, 378-380 (2009)

6. Bernevig, B. A., Hughes, T. L. \& Zhang, S. C. Quantum spin Hall effect and topological phase transition in HgTe quantum wells. Science 314, 1757-1761 (2006).

7. Qi, X. L., Hughes, T. L. \& Zhang, S. C. Fractional charge and quantized current in the quantum spin Hall state. Nat. Phys. 4, 273-276 (2008).

8. Li, R. D. et al. Dynamical axion field in topological magnetic insulators. Nat. Phys. 6, 284-288 (2010).

9. Qi, X. L. et al. Inducing a magnetic monopole with topological surface states. Science 323, 1184-1187 (2009).

10. Fu, L. \& Kane, C. L. Superconducting proximity effect and Majorana fermions at the surface of a topological insulator. Phys. Rev. Lett. 100, 096407 (2008).

11. Fu, L. \& Berg, E. Odd-parity topological superconductors: theory and application to $\mathrm{Cu}_{x} \mathrm{Bi}_{2} \mathrm{Se}_{3}$. Phys. Rev. Lett. 105, 097001 (2010).

12. Yu, R. et al. Quantized anomalous Hall effect in magnetic topological insulators. Science 329, 61-64 (2010).

13. Konig, M. et al. Quantum spin hall insulator state in HgTe quantum wells. Science 318, 766-770 (2007).

14. Brune, C. et al. Quantum Hall effect from the topological surface states of strained bulk HgTe. Phys. Rev. Lett. 106, 126803 (2011).

15. Fu, L., Kane, C. L. \& Mele, E. J. Topological insulators in three dimensions. Phys. Rev. Lett. 98, 106803 (2007).

16. Zhang, H. J. et al. Topological insulators in $\mathrm{Bi}_{2} \mathrm{Se}_{3}, \mathrm{Bi}_{2} \mathrm{Te}_{3}$ and $\mathrm{Sb}_{2} \mathrm{Te}_{3}$ with a single Dirac cone on the surface. Nat. Phys. 5, 438-442 (2009)

17. Xia, Y. et al. Observation of a large-gap topological-insulator class with a single Dirac cone on the surface. Nat. Phys. 5, 398-402 (2009).

18. Chen, Y. L. et al. Experimental realization of a three-dimensional topological insulator, $\mathrm{Bi}_{2} \mathrm{Te}_{3}$. Science 325, 178-181 (2009).

19. Qu, D. X. et al. Quantum oscillations and Hall anomaly of surface states in the topological insulator $\mathrm{Bi}_{2} \mathrm{Te}_{3}$. Science 329, 821-824 (2010).

20. Butch, N. P. et al. Strong surface scattering in ultrahigh-mobility $\mathrm{Bi}_{2} \mathrm{Se}_{3}$ topological insulator crystals. Phys. Rev. B 81, 241301 (2010).

21. Taskin, A. A. et al. Observation of Dirac holes and electrons in a topological insulator. Phys. Rev. Lett. 107, 016801 (2011).

22. Analytis, J. G. et al. Two-dimensional surface state in the quantum limit of a topological insulator. Nat. Phys. 6, 960-964 (2010).

23. Ren, Z. et al. Observations of two-dimensional quantum oscillations and ambipolar transport in the topological insulator $\mathrm{Bi}_{2} \mathrm{Se}_{3}$ achieved by $\mathrm{Cd}$ doping Phys. Rev. B 84, 075316 (2011).

24. Hor, Y. S. et al. p-type $\mathrm{Bi}_{2} \mathrm{Se}_{3}$ for topological insulator and low-temperature thermoelectric applications. Phys. Rev. B 79, 195208 (2009).

25. Chen, C. Y. et al. Robustness of topological order and formation of quantum well states in topological insulators exposed to ambient environment. Proc. Natl. Acad. Sci. U.S.A. 109, 3694-3698 (2012).

26. Kim, D. et al. Intrinsic electron-phonon resistivity of $\mathrm{Bi}_{2} \mathrm{Se}_{3}$ in the topological regime. Phys. Rev. Lett. 109, 166801 (2012).

27. Novoselov, K. S. et al. Two-dimensional gas of massless Dirac fermions in graphene. Nature 438, 197-200 (2005).

28. Zhang, Y. B. et al. Experimental observation of the quantum Hall effect and Berry's phase in graphene. Nature 438, 201-204 (2005)
29. Bolotin, K. I. et al. Ultrahigh electron mobility in suspended graphene. Solid State Commun. 146, 351-355 (2008).

30. Grimvall, G. The Electron-Phonon Interction in Metals. (North-Holland, 1981)

31. Damascelli, A., Hussain, Z. \& Shen, Z. X. Angle-resolved photoemission studies of the cuprate superconductors. Rev. Mod. Phys. 75, 473-541 (2003).

32. Zhou, X. J., Cuk, T., Devereaux, T., Nagaosa, N. \& Shen, Z. X. Handbook of High Temperature Superconductivity, edited by Schrieffer, J. R. (Springer, Berlin, 2006), Chap. 3.

33. Hatch, R. C. et al. Stability of the $\mathrm{Bi}_{2} \mathrm{Se}_{3}$ (111) topological state: Electron-phonon and electron-defect scattering. Phys. Rev. B 83, 241303 (2011).

34. Pan, Z. H. et al. Measurement of an exceptionally weak electron-phonon coupling on the surface of the topological insulator $\mathrm{Bi}_{2} \mathrm{Se}_{3}$ using angle-resolved photoemission spectroscopy. Phys. Rev. Lett. 108, 187001 (2012).

35. Zhu, X. T. et al. Interaction of phonons and Dirac fermions on the surface of $\mathrm{Bi}_{2} \mathrm{Se}_{3}$ : a strong kohn anomaly. Phys. Rev. Lett. 107, 186102 (2011).

36. Liu, G. D. et al. Development of a vacuum ultraviolet laser-based angle-resolved photoemission system with a superhigh energy resolution better than $1 \mathrm{meV}$. Rev. Sci. Instrum. 79, 023105 (2008)

37. Valla, T., Fedorov, A. V., Johnson, P. D. \& Hulbert, S. L. Many-body effects in angle-resolved photoemission: quasiparticle energy and lifetime of a Mo(110) surface state. Phys. Rev. Lett. 83, 2085 (1999).

38. LaShell, S., Jensen, E. \& Balasubramanian, T. Nonquasiparticle structure in the photoemission spectra from the $\mathrm{Be}(0001)$ surface and determination of the electron self energy. Phys. Rev. B 61, 2371 (2000).

39. Cheng, W. \& Ren, S. F. Phonons of single quintuple $\mathrm{Bi}_{2} \mathrm{Te}_{3}$ and $\mathrm{Bi}_{2} \mathrm{Se}_{3}$ films and bulk materials. Phys. Rev. B 83, 094301 (2011).

40. Richter, W. \& Becker, C. R. A Raman and far-infrared investigation of phonons in the rhombohedral $\mathrm{V}_{2}-\mathrm{VI}_{3}$ compounds $\mathrm{Bi}_{2} \mathrm{Te}_{3}, \mathrm{Bi}_{2} \mathrm{Se}_{3}, \mathrm{Sb}_{2} \mathrm{Te}_{3}$ and $\mathrm{Bi}_{2}\left(\mathrm{Te}_{1-\mathrm{x}} \mathrm{Se}_{\mathrm{x}}\right)_{3}$ $(0<\mathrm{x}<1),\left(\mathrm{Bi}_{1-\mathrm{y}} \mathrm{Sb}_{\mathrm{y}}\right)_{2} \mathrm{Te}_{3}(0<\mathrm{y}<1)$. Phys. Stat. Sol. (b) 84, 619-628 (1977).

41. Huang, G. Q. Surface lattice vibration and electron-phonon interaction in topological insulator $\mathrm{Bi}_{2} \mathrm{Te}_{3}$ (111) films from first principles. EPL 100, 17001 (2012)

\section{Acknowledgments}

We thank Mr. Yuan Pang and Prof. Li Lu for providing us n-type $\mathrm{Bi}_{2} \mathrm{Te}_{3}$ single crystals, Prof. Liling Sun and Prof. Zhong-xian Zhao for their help in the characterization of the samples. We thank Mr. Quansheng Wu, Dr. Hongming Weng, Prof. Xi Dai and Prof. Zhong Fang for helpful discussions. This work is supported by the National Natural Science Foundation of China (91021006) and the Ministry of Science and Technology of China (973 program 2011 CB921703 and 2013CB921700).

\section{Author contributions}

C.Y.C. and X.J.Z. conceived and designed the research; C.Y.C. performed measurements Z.J.X., Y.F., H.M.Y., A.J.L., S.L.H., D.X.M., J.F.H., Y.Y.P., LX.L., Y.L., L.Z., G.D.L., X.L.D., J.Z., L.Y., X.Y.W., Q.J.P., Z.M.W., S.J.Z., F.Y., C.T.C., Z.Y.X. and X.J.Z. contributed new reagents/analytic tools; C.Y.C. and X.J.Z. analyzed data and wrote the paper.

\section{Additional information}

Supplementary information accompanies this paper at http://www.nature.com/ scientificreports

Competing financial interests: The authors declare no competing financial interests.

How to cite this article: Chen, C.Y. et al. Tunable Dirac Fermion Dynamics in Topological Insulators. Sci. Rep. 3, 2411; DOI:10.1038/srep02411 (2013).

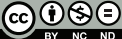

This work is licensed under a Creative Commons AttributionNonCommercial-NoDerivs 3.0 Unported license. To view a copy of this license, visit http://creativecommons.org/licenses/by-nc-nd/3.0 